\title{
Birefringence Control in Silicon Wire Waveguide by Using Over-etch
}

\author{
Ika Puspita*, A. M. Hatta \\ Photonics Engineering Laboratory, Department of Engineering Physics, Institut Teknologi Sepuluh \\ Nopember \\ e-mail: ika.tf10@gmail.com
}

\begin{abstract}
Silicon wire waveguide technology becomes great issue in optical communication system. The high index contrast of the silicon wire waveguide induced the birefringence. It played important role in silicon wire waveguide loss since it caused polarization dependent loss (PDL), polarization mode dispersion (PMD) and wavelength shifting. Hence, controlling birefringence in silicon wire waveguide become very important. The current birefringence controlling techniques by using cladding stress and geometrical variation in bulk silicon waveguide was presented. Unfortunately, it could not obtain zero birefringence when applied to silicon wire waveguide. The over-etching technique was employed in this paper to obtain zero birefringence. The tall silicon wire waveguide obtained minimum birefringence
\end{abstract}

Keywords: Birefringence control, silicon wire waveguide, over-etching

\section{Introduction}

Silicon wire waveguide structure was fabricated by depositing the silica layer onto the silicon using plasma-enhanced chemical vapor deposition (PECVD). The deposition process involves temperature of $300 \mathrm{o} \mathrm{C}$. Silicon wire waveguide has high refractive index contrast $(\Delta \sim$ $40 \%$ ). It may lead to birefringence [1]. The presence of birefringence is an important issue in silicon wire waveguide because it can induce polarization mode dispersion (PMD), polarization dependent loss (PDL) and wavelength shifting [2-4]. In silicon wire waveguide, the birefringence is caused by the anisotropic distribution of the stresses induced by thermal coefficient mismatch and lattice defect between core and cladding material during deposition process [5-7].

The birefringence controlling in large cross-section silicon on insulator (SOI) has been reported [8-10]. In [8,9], stress engineering was employed to control birefringence in an Array Waveguide Grating (AWG) by varying upper cladding thickness. The birefringence was also controlled by changing the silicon waveguide geometry, especially the ridge width. Under stress condition, silicon waveguide would have zero birefringence in a wider ridge width [10].

The birefringence in highly-contrast waveguide such as silicon-on-insulator (SOI) waveguide and silicon wire waveguide is largerly dominated by geometrical birefringence [11]. In the other hand, the birefringence in waveguides with over-etch has been studied in $[11,12]$. The purpose of over-etching process is to create a pedestal. The pedestal could modify the stress distribution in the core [12]. However, the silicon wire waveguide with over-etching has not been studied yet. In this paper, the effect of over-etching to control birefringence in silicon wire waveguide has been studied theoretically and numerically. Since, the dominant birefringence in silicon wire waveguide is geometrical birefringence, the stress birefringence from thermal stress can be neglected.

\section{Research Method}

Figure 1(a) shows the silicon wire waveguide structure. The waveguide core, typically of $250 \mathrm{~nm}$ or $300 \mathrm{~nm}$ on thickness and formed from a silicon layer, through photolitography process and etched down to the lower cladding. However, the core dimension was varied to know the each effect of the dimension. The upper cladding is silicon nitride (Si3N4) with $1 \mu \mathrm{m}$ in thickness. While the lower cladding is silicon dioxide (SiO2) with $3 \mu \mathrm{m}$ in thickness. The refractive index of silicon, silicon nitride, and silicon dioxide in wavelength of $1550 \mathrm{~nm}$ are 3.444 , 2.4629 , and 1.444 respectively. The numerical approach used Finite Difference Method (FDM) 
that was performed by using GUI Octave software. The method in this paper divided in four main following steps.

a. Step 1: The core thickness was set fixed. The core width and The over-etched depth would be varied. This step as means to know the effect of the core thickness and overetched depth to the guided mode in the core region.

b. Step 2: The core width was set fixed. The core thickness and the over-etched depth would be varied. This step as means to know the effect of core thickness and overetched depth to the guided mode in the core region.

c. Step 3: As the result of the step 1 and step 2, the possible core dimension for guiding the mode was used to do numerical approach of birefringence. This step is used to obtain the smallest birefringence. Figure 1(b) shows the over-etched waveguide.

d. Step 4: The final step is validation by comparing the result in step 3 to the published data. This step is used to know the validity of the over-etched silicon wire waveguide numerical approach.

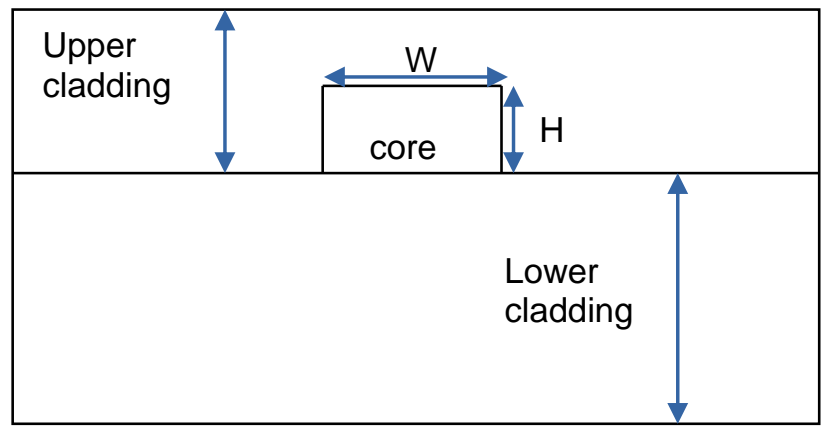

Figure 1(a). Silicon wire waveguide cross-section

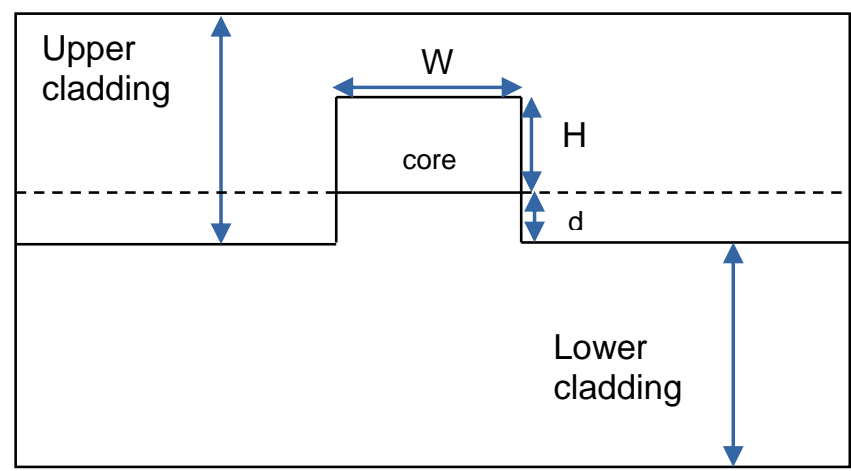

Figure 1(b). Over-etched silicon wire waveguide

\section{Birefringence in over-etched silicon wire waveguide}

In silicon wire waveguide without over-etch, the core aspect ratio is defined as,

$$
a=\frac{H}{W}
$$

while, the over-etching process in a silicon wire waveguide creates a pedestal that wil modify the aspect ratio of the core region. The over-etching can be assumed as the change of the aspect ratio of the core accompanied by the a change in material composition (as the core material differs from the pedestal material). The aspect ratio of core is redefined as,

$$
b=\frac{(H+d)}{W}
$$


As change in core aspect ratio and also the compition, the effective index of $x$ and $y$ direction could be change. It induced the change in number of guide mode and birefringence. The birefringence is defined as the difference between effective index of TM and TE polarized fundamental modes [13].

$$
B=n_{e f f} T M-n_{e f f} T E
$$

The total birefringence in over-etched silicon wire waveguide can be approximated as the sum of the geometrical birefringence and the over-etched birefringence:

$$
B_{\text {total }}=B_{\text {geo }}+B_{\text {over }}
$$

where the geometrical birefringence, defined as the difference in effective indexes corresponding to solutions of the scalar wave equation. The geometrical birefringence is stressfree birefringence. Since the thermal stress was neglected, hence the over-etching birefringence defined as the difference in effective indexes of fundamental mode corresponding to solutions of the wave equation, with the boundary condition as a function of over-etched depth [14].

$$
B_{\text {over }}=2 n_{\text {core }} \sin \left(\theta_{2}-\theta_{1}\right) \cos \left(\theta_{1}-\theta_{2}\right)
$$

where the $\theta_{1}$ and $\theta_{2}$ can be obtained by using eigen value equation of TE and TM modes as follows,

TE Eigen value equation

$$
\left[\mathrm{k}_{o} \mathrm{n}_{\text {core }}(h+d) \cos \theta_{1}-m \pi\right]=\tan ^{-1}\left[\frac{\sqrt{\sin ^{2} \theta_{1}-\left(\frac{n_{l c}}{n_{\text {core }}}\right)^{2}}}{\cos \theta_{1}}\right]+\tan ^{-1}\left[\frac{\sqrt{\sin ^{2} \theta_{1}-\left(\frac{n_{u c}}{n_{\text {core }}}\right)^{2}}}{\cos \theta_{1}}\right]
$$

TM Eigen value equation

$$
\mathrm{k}_{o} \mathrm{n}_{e f f x} w \cos \theta_{2}=2 \tan ^{-1}\left[\frac{\sqrt{\left(\frac{n_{e f f x}}{n_{u c}}\right)^{2} \sin ^{2} \theta_{2}-1}}{\left(\frac{n_{e f f x}}{n_{u c}}\right) \cos \theta_{2}}\right]
$$

\section{Result and Analysis}

Silicon wire waveguide typically fabricated in thickness of $250 \mathrm{~nm}$ or $300 \mathrm{~nm}$. Table 1 and 2 show the numerical result of the effective index calculation with the width variation.

Table 1. Effective index calculation for thickness of $250 \mathrm{~nm}$

\begin{tabular}{ccc} 
width & TE & TM \\
\hline 300 & 2.530162 & 2.448033 \\
350 & 2.590297 & 2.470601 \\
400 & 2.644973 & 2.492485 \\
450 & 2.69178 & 2.512629 \\
\hline
\end{tabular}

Table 2. Effective index calculation for thickness of $300 \mathrm{~nm}$

\begin{tabular}{ccc}
\hline width & TE & TM \\
\hline 150 & 2.419512 & 2.416048 \\
200 & 2.457573 & 2.446684 \\
250 & 2.520665 & 2.486742 \\
300 & 2.594384 & 2.52845 \\
\hline
\end{tabular}


From the table above, the silicon wire waveguide with the thickness of $300 \mathrm{~nm}$ has lower differences in TE and TM effective indexes. This thickness was used to do step 1. The width of $350 \mathrm{~nm}$ was used to do step 2.

Figure 2 shows the numerical result of step 1. The core thickness was fixed at $300 \mathrm{~nm}$ and the width was varied from $150-350 \mathrm{~nm}$. The over-etched depth effect added to the calculation. The over-etched depth was varied from $0-200 \mathrm{~nm}$. The upper and lower cladding thickness were fixed at $1000 \mathrm{~nm}$ and $3000 \mathrm{~nm}$ respectively.

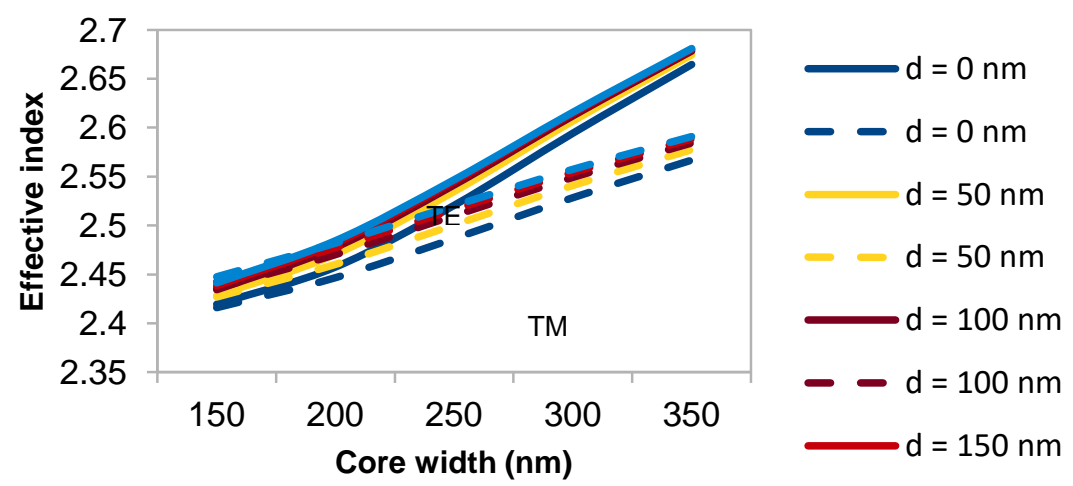

Figure 2. Effective index calculation for step 1

For core thickness range of $150-200 \mathrm{~nm}$, no mode guided in the core region. While, for core thickness range of $200-350 \mathrm{~nm}$, both TE and TM mode guided in the core region. This result shows that the waveguide with the core width $200 \mathrm{~nm}$ and over-etched depth of $200 \mathrm{~nm}$ has birefringence of -00253 .

Figure 3 shows the numerical approach result of over-etched silicon wire waveguide. The core width was fixed at $350 \mathrm{~nm}$ and the thickness was varied from $220-300 \mathrm{~nm}$. The overetched depth was varied from $0-200 \mathrm{~nm}$. The upper and lower cladding thickness were fixed at $1000 \mathrm{~nm}$ and $3000 \mathrm{~nm}$ respectively

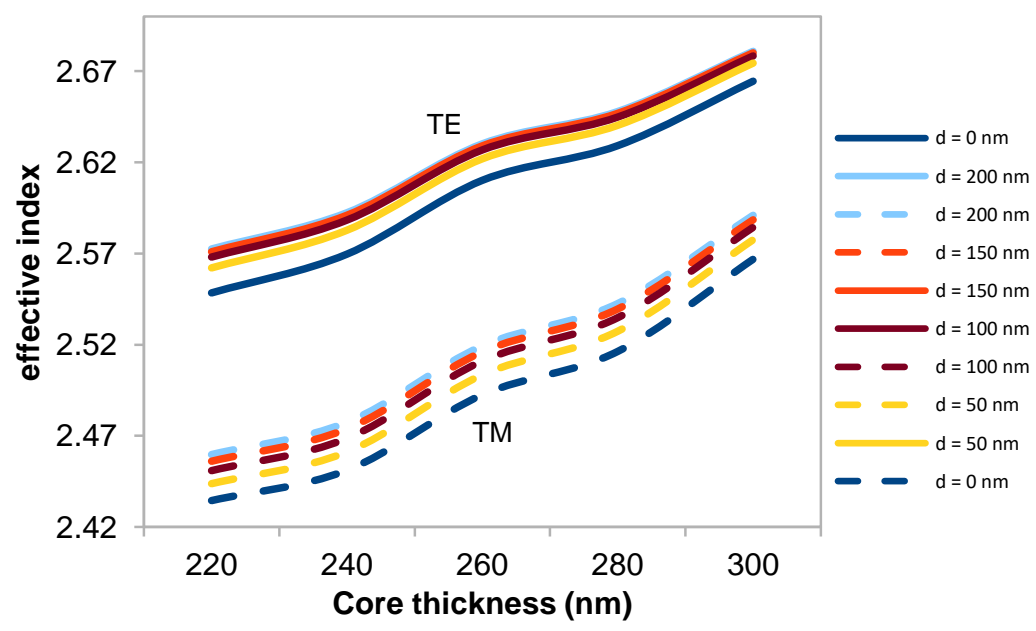

Figure 3. Effective index calculation for step 2

For core thickness range of $220-240 \mathrm{~nm}$, only TE mode guided in the core region. After over-etching process, both TE and TM mode could be guided in the core region. While, for 
core thickness range of $240-300 \mathrm{~nm}$, both TE and TM mode guided in the core. The result shows that over-etching process pulls the mode to the confined part.

From table1, without over-etching, the core width and thickness that possible to guide modes are at $350 \mathrm{~nm}$ and $250 \mathrm{~nm}$, respectively. Then the effect of over-etched depth added to the numerical calculation of birefringence. The over-etched depth was varied from $0-225 \mathrm{~nm}$. The upper and lower cladding thickness were fixed at $1000 \mathrm{~nm}$ and $3000 \mathrm{~nm}$ respectively. The result shows in Figure 4.

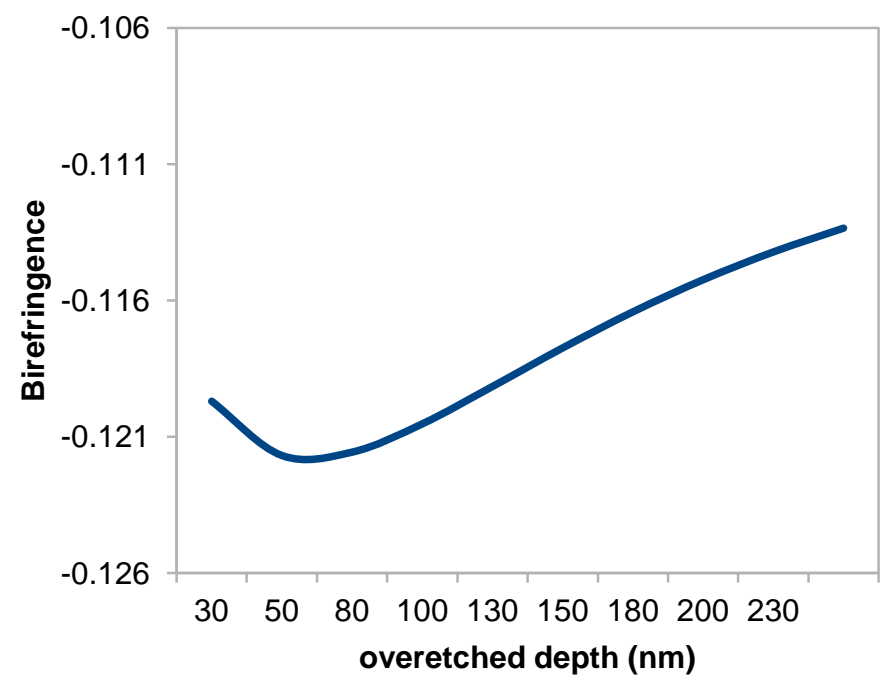

(a)

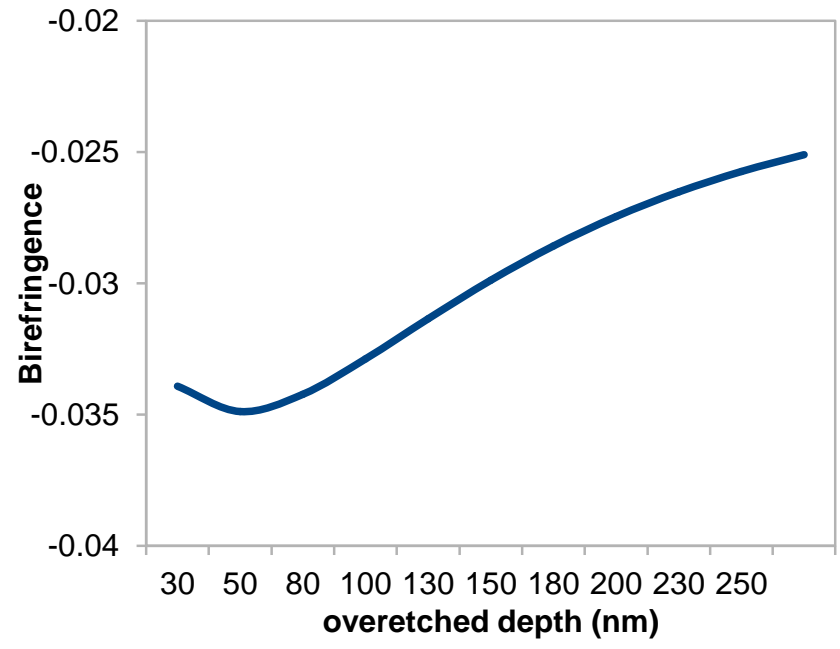

Figure 4. birefringence calculation of (a) $350 \mathrm{~nm}$ in width and 250 in thickness (b) 250 in width and $300 \mathrm{~nm}$ in thickness

Figure 4(b) shows the numerical result of the over-etched silicon wire waveguide with the core width and thickness was fixed at $250 \mathrm{~nm}$ and $300 \mathrm{~nm}$, respectively. The over-etched depth was varied from $0-225 \mathrm{~nm}$. The upper and lower cladding thickness were fixed at 1000 $\mathrm{nm}$ and $3000 \mathrm{~nm}$ respectively.

The small birefringence value could be obtained by changing the core width to be narrower. The adding of over-etched part would pull the non-guided mode to the confined part. The tall rectangular core dimension has lower birefringence than the wide one. 


\section{Conclusion}

The effect of over-etching in silicon wire waveguide has been studied theoretically and numerically. The thermal stress effect to the waveguide was neglected since the dominant birefringence is geometrical birefringence. Without over-etched the tall waveguide core has lower birefringence than the wider one. The ove-etching pull the unguided mode to the confined region and minimize the birefringence in silicon wire waveguide. The smallest birefringence is 0.00253 . It could be obtained by the waveguide with the core dimension of $200 \mathrm{~nm}$ in width and $300 \mathrm{~nm}$ in thickness. The given over-etched depth is $200 \mathrm{~nm}$.

\section{Acknoledgement}

This paper work is supported by ITS Fresh graduate Student Scholarship from Indonesia Goverment.

\section{References}

[1] H Yamada, T Chu, S Ishida, Y Arakawa. Si photonic wire waveguide devices. IEEE J. Quantum Elect. 2006; 12(6): 2006: 1371-1379.

[2] JN Damask. Polarization optics in telecommunication, Chapter 8, New York: Springer. 2005: 297 383.

[3] M Huang and $X$ Yan. Thermal-stress effects on the temperature sensitivity of optical waveguides. $J$. Opt. Soc. Am. B. 2003; 20(6): 1326-1333.

[4] S Janz, P Cheben, $\mathrm{H}$ Dayan, and R Deakos. Measurement of birefringence in thin-film waveguides by Rayleigh scattering. Opt. Lett. 2003; 28(19): 1778-1780.

[5] Huang M. Stresses effect on the performance of optical waveguide. Int. J. Sol. and Struct. 2003; 40: $1615-1632$.

[6] Huang M. Thermal stresses in optical waveguide. Opt. Lett. 2003; 28(23): 2327-2329.

[7] WD Callister. Material Science and Engineering: An Introduction Seventh Ed, Chapter 6, pp. 137 139, USA: John Wiley and Sons Inc, 2007.

[8] WN Ye, et.al. Stress-induced birefringence in silicon-on-insulator (SOI) waveguides. Opt.elect Integrat on Si. proc. SPIE. 2004; 5357: 57-66.

[9] J Lockwood and L Pavesi, Silicon photonics II component an7d integration. Springer: Heidelberg. $2010 ; 119$.

[10] $\mathrm{DX} \mathrm{Xu}$, et.al. Eliminating the birefringence in silicon-on-insulator ridge waveguide by use of cladding stress. Opt. Lett. 2004; 29(20): 2384-2386.

[11] Dumais P. Thermal stress birefringence in buried-core wavguide with over-etch. IEEE J. Quant. Elect. 2011; 47(7): 989 - 996.

[12] Dumais P. Modal birefringence analysis of strained buried-core waveguides. J Lightwave Tech. 2015; 30(6): 906-912.

[13] K Okamoto, Fundamental of Optical Waveguides, Academic Press, USA, 2000.

[14] Schriemer HP. Modal birefringence amd power density distribution in strained buried-core square waveguides. EEE J. Quant. Elect. 2004; 40(8): 1131 - 1139. 